\title{
Common Components of Home-Based Family Therapy Models: The HBFT Partnership in Kansas
}

\section{R. Macchi}

Marriage and Family Therapy Program, School of Family Studies and Human Services, Kansas State University, 109 Campus Creek Complex, Manhattan, KS 66506, USA. Email: crmacchi@ksu.edu

\section{Nancy O'Conner}

Marriage and Family Therapy Program, School of Family Studies and Human Services, Kansas State University, 104 Campus Creek Complex, Manhattan, KS 66506, USA. Email: noconner@ksu.edu

This paper published as: C. R. Macchi and Nancy O'Conner (2010). Common Components of Home-Based Family Therapy Models: The HBFT Partnership in Kansas. Contemporary Family Therapy, Volume 32, Number 4, Pages 444-458. 


\section{Abstract}

In this article, we provide an analysis of components shared by varying home-based family therapy (HBFT) practices and evidence-based models. Applying a consistent statewide standard for HBFT presents challenges for the training of therapists from varying disciplines, with different levels of experience, and from diverse agencies. We propose focusing on common components across existing evidence-based models and discuss the trainings developed to integrate those components. We address indications and contraindications for using an HBFT approach and illustrate ways to address the particular challenges of HBFT supported by a unique partnership in Kansas. We conclude by offering suggestions for further research and for continued training development.

Keywords: Home-based family therapy; Training; Evidence-based practice

\section{Introduction}

Clinicians have employed home-based family therapy (HBFT) practices since the 1980s. Whereas previous programs had emphasized individuals at risk, with the establishment in 1993 of the Family Preservation and Family Support Services Program communities began to acknowledge the larger systemic nature of a child's mental health and his or her behavior within the context of the family (United States Congress 1993).

The family preservation model is based on a preference for leaving a child in the home while empowering families to be actively engaged in the therapeutic process. The model assumes that a child who is safe from neglect and abuse will fare better in the home than will one placed outside of the home. This preference provides the least restrictive environment and an opportunity for therapists and other human service workers to address issues affecting the whole family (Christensen 1995; Cortes 2004). In this article, we address the specific types of training needed to prepare clinicians for effectively delivering HBFT. 
A review of the HBFT-related literature to date reveals the use of several theoretical frameworks: a social ecological model, social learning theory, cognitive behavioral therapy (CBT), general family systems, functional family therapy, multisystemic therapy, structural-strategic family therapy, solution-focused family therapy, and family psychoeducation (Berg 1994; Boyd-Franklin and Bry 2000; Henggeler and Lee 2003; Lindblad-Goldberg et al. 1998; Sexton and Alexander 2000). Despite the use of diverse theoretical frameworks, models informing HBFT address five common components relevant to clinical practice in a family's home. These components include the environment and context, the family's roles and expectations, the therapist's roles and expectations, the therapeutic relationship, and the focus of clinical work. Each component influences the other components and the progress of therapy.

A social ecological lens (Bronfenbrenner 1979) provides a focus on the environmental and contextual components impacting the family and the therapy process. This lens broadens the scope beyond individual family members and the family as a whole. Consideration is given to physical and cultural contexts including, but not limited to the following: the family home, neighborhood, local and state agency policies and involvement, ethnicity, socio-economic status, and treatment history. A family's current and intergenerational experiences within those contexts influence the members' expectations of the therapy process and outcomes.

The therapeutic relationship between the therapist and the family members is defined and refined throughout the therapy process. However, joining, assessment, and contracting influence the trajectory of that relationship. The therapeutic relationship within the therapy office begins with the family accommodating to the therapist's familiarity with the clinical context. By contrast, in the family's home, that relationship begins with the therapist as the guest accommodating to the family's familiar environment. Cottrell (1994) describes varying degrees of anxiety a therapist may experience while visiting the home. Issues of safety, perceived lack of control over the environment, and the distances traveled to get to the family's home also produce a degree of anxiety, stress, and even burnout that the therapist must manage effectively to engage in the work while in the home (Adams and Maynard 2000; Christensen 1995). 
Several mental health disciplines address the importance of acquiring a set of core competencies or meeting standards necessary for providing effective clinical treatment (American Counseling Association 2005; National Association of Social Workers 2000; Nelson et al. 2007; Rodolfa 2009). However, none of the disciplines describes the competencies uniquely associated with using an HBFT approach. Training programs generally focus on preparing clinicians to provide therapeutic services in office-based settings (Christensen 1995). HBFT therapists attempting to develop those competencies are often challenged with obtaining training after graduate school while they attempt to meet the competing demands of high caseloads and other agency responsibilities. Additional challenges arise as they attempt to apply their knowledge of evidence-based treatments while simultaneously adhering to both the protocols of manualized treatments as well as their agencies' policies, procedures, and expectations. Adaptations of an evidence-based model aimed at meeting competing expectations potentially undermine the principles of the model. Developing a state-wide training program that can be effectively applied across agencies, geographic locations, and varying client populations requires an overarching framework rooted in the evidence while also adaptable to unique contexts.

\section{The HBFT Partnership}

In 2005, the Social and Rehabilitative Services of Kansas requested proposals from several educational institutions to provide training for the HBFTs throughout Kansas. A training contract for the HBFT Partnership was established with Kansas State University to provide HBFT therapists with the training needed to become credentialed and, therefore, eligible for Medicaid reimbursement. The Kansas partners include Kansas State University, the Kansas Department of Social and Rehabilitative Services, 28 community mental health centers, and Kansas Health Solutions, a managed care organization that oversees mental health services provided through Medicaid funding.

Since the initial contract, beginning in 2006, the HBFT Partnership has provided 535 therapists in Kansas with the trainings necessary for credentialing to obtain Medicaid reimbursement. Through annual contracts, the HBFT Partnership provides practitioners with the necessary resources and training for using evidence-based practices to evaluate the process and impact of their home-based 
work with the families they serve. Currently, 715 clinicians from various agencies are registered with the partnership, including 267 from 29 community mental health centers, 197 from 5 private contractors, and 41 private practitioners. The combined work of these practitioners serves clients in all of the 105 counties of Kansas.

\section{Mission and Objectives}

The mission of the HBFT Partnership is to provide an approach to training clinicians that addresses two priorities: explore and apply the best principles of current evidence-based practices while evaluating the impact resulting from participation in the training components on the clinicians' practice. The components are designed to support HBFT clinical practice. The objectives and principles guiding the development of each component include providing focused knowledge and skill development, prioritizing the use of supervision, facilitating therapist self-care strategies, providing multiple opportunities for ongoing therapist support and collegial relationships, and encouraging therapist collaborations and consultations.

\section{Components of the HBFT Partnership}

The training components are designed to facilitate the mission and objectives of the HBFT Partnership. The components include core trainings, videoconferences, and a tailored website including online learning modules and CD learning companions. Participants involved in each of the contract components also receive continuing education credits needed for maintaining state clinical licensure with the Kansas Behavioral Sciences Regulatory Board.

Core Training

The Core Training is a one-day, in-person experience annually offered at three different sites throughout the state of Kansas. Therapist participation in the Core Training meets the criteria for reimbursement of HBFT therapy services set by the Kansas Department of Social and Rehabilitation Services, Division of Disability and Mental Health Services (SRS), and the Centers for Medicare and Medicaid Services (CMS). To-date, 535 therapists have participated in 16 Core Trainings over the past 4 years. Wasik and Roberts (1994) note, 
Ongoing training and supervision may be especially important for home visitors because home visiting is a difficult, stressful, and at times, lonely position.... Project directors should consider group training, where sharing of information among home visitors is possible and opportunities are available to learn not only from trainers and supervisors, but also from other home visitors. (p. 340)

Therefore, the training reviews the principles of evidence-based HBFT practices while providing several experiential opportunities for therapists to share ways to connect the evidence-based principles with specific strategies and techniques for conducting culturally competent HBFT. Integrated throughout the training are specific strategies necessary for prioritizing therapist selfcare and use of supervision to reduce the stress and potential burnout that is often associated with providing varying types of therapy (Bakker and Schaufeli 2001; Carroll et al. 1999). Use of the Professional Quality of Life Scale (ProQOL R-IV, (Stamm 2002) provides a measure for therapists to assess personal levels of compassion satisfaction, compassion fatigue, and burnout.

Videoconferences

The videoconferences address diagnoses listed in the Diagnostic and Statistical Manual of Mental Disorders (American Psychological Association, DSM-IV-TR 2000) as well as ethical issues specifically associated with the practice of HBFT. Offered from the university site, 158 clinicians have participated at each of eight different locations via videoconference during eight trainings. The availability of multiple sites minimizes the distance clinicians must travel to attend, and the videoconferencing capability enables participants an opportunity to interact with and develop collegial relationships with others around the state. Figley (2002) explained,

The evidence that regional workers are more at risk than workers in major cities is probably linked [with therapists' experience of secondary trauma].... This has implications for organization and deployment of resources, especially in rural areas. The utilization of technology such as video conferencing to 
enable rural case managers [and HBFT therapists] to discuss stress-related issues with other professionals could be expected to reduce isolation. (p. 101)

Videoconferences have addressed topics such as Families of a Child with an Autism Spectrum Disorder, HBFT Treatment of Children with PTSD and Depression, Ethical Implications of Therapist Self-Care, and Ethical Implications of Collaboration in HBFT.

Website, Online Learning Modules, and CD Learning Companions

The HBFT Partnership website has been designed to provide HBFT therapists with ongoing training and support, opportunities for connecting and sharing with colleagues, and access to the latest HBFT resources in varied, accessible formats. The website includes a Discussion Board and a Resources Section that are located behind a gated access available to all registered users. HBFT Partners can access each either directly from the website home page or through the online learning modules. The Discussion Board provides a place for clinicians to discuss challenges of providing HBFT and share resources with one another. The Resources Section includes articles, websites, and other documents referenced throughout each of the training components. A Therapists Spotlight section, located on the homepage, is regularly updated to highlight the work of an HBFT therapist, including personal reflections on his or her development and application of skills that have improved the effectiveness of his or her service delivery. The website components provide HBFT clinicians with multiple opportunities to reduce the isolation experienced while expanding opportunities for obtaining helpful resources and developing clinical skills.

The online learning modules are designed to provide advanced training in four different areas: Therapeutic Skills, Family Issues, Therapist Self-Care, and Supervision. Modules address therapeutic skills most relevant to HBFT, including crisis management, cultural competency, and safety training. Those focusing on family issues train clinicians to work with families who have a member with a chronic medical condition and with stepfamilies. Modules on therapist self-care address unique HBFT factors that influence clinicians' ability to manage anxiety, prevent burnout, and balance their personal and professional lives. A supervision module explores ways therapists 
can effectively use supervision to manage appropriate boundaries between their personal and professional lives, access available resources, receive guidance and support, and simultaneously maintain varying points of view in their work with clients. Through the most recent contract, CDs were developed to enable therapists to listen to the content of three of the modules while they travel to and from families' homes. When clinicians return to the office after listening to the $\mathrm{CD}$, they are able to log onto the module, review content they would like to explore in greater depth, access supporting literature, and participate in discussion threads. Lastly, they complete a post-test to signify their completion of the module and evaluate their understanding of the material.

Providing a statewide training plan requires an increased understanding of the common components shared by the varying models of HBFT. This understanding informs the development and delivery of training to and support of HBFT therapists. Each of the training opportunities is designed to address the common components of the current evidenced-based models of HBFT.

\section{Common Components of HBFT Evidence-Based Practice}

There are a few widely known and well-established evidence-based, home-based, family therapy, family preservation, and community-based approaches. These approaches are designed to work with specific client family populations where a child is at risk for out of home placement. Functional family therapy (FFT) has been applied to work with families with a youth who is at risk for delinquency, violent behaviors, and drug use (Sexton and Alexander 2000); multisystemic therapy (MST) has guided work with youth exhibiting antisocial behaviors and substance abuse (Henggeler and Lee 2003; Lindblad-Goldberg et al. 1998; Saldana and Sheidow 2005); homebuilders' intensive family preservation and reunification services (IFPS) has focused on therapy with families who have a child experiencing problems with delinquency and drug use (Fraser and Haapala 1987; Institute for Family Development 2005); and the Oregon multidimensional treatment foster care model (MTFC) has informed work with children and adolescents exhibiting serious behavioral issues and their families (Chamberlain and Smith 2003; National Institute of Mental Health 2007). Adherents of these approaches work with families addressing the issues that lead to their involvement in the child welfare and juvenile justice systems. Each model is based on a common assumption that community-based support provides 
the family with the most immediate, accessible, and generalizable treatment.

Each model describes a therapeutic process that is time-intensive for the family and the therapist; collaborations occur among the therapist, family members, clinical supervisors, and other professionals within the community; and treatments facilitate family change over a relatively brief period. The evidence-based treatments use standardized approaches that are responsive to the distinct features of the family and the issues they are currently facing. Because HBFT is demanding work, evidence-based models suggest that therapists carry limited caseloads, allowing the therapist to make longer and more frequent visits with families. Furthermore, the demands on the therapist necessitate continuous and consistent administrative and supervisory support. Despite subtle differences between the models, each addresses similar components including the environment and context, the family roles and expectations, the therapist roles and expectations, the therapeutic relationship, and the goals of clinical work. Figure 1 illustrates the interlocking properties of each component. While an HBFT component reflects one aspect of the work, each component influences the other components in several ways. In the next sections we examine the components and the varying ways the HBFT Partnership developed an integrated approach to training and support that address each component.

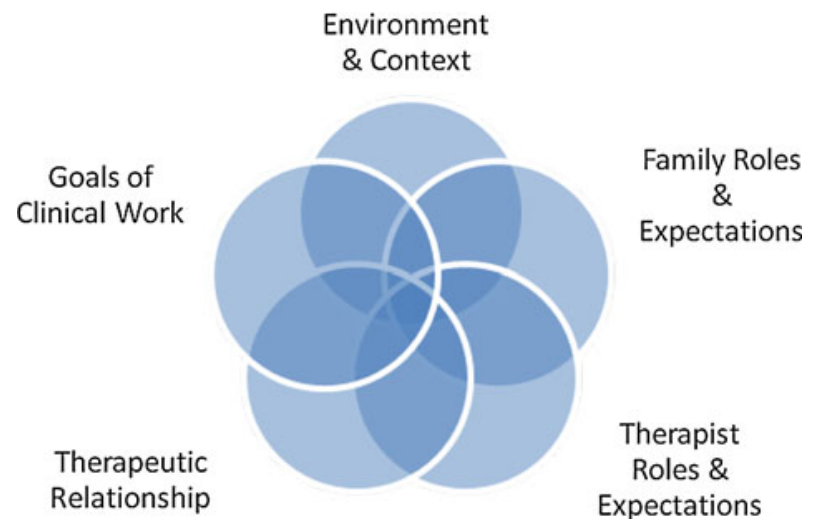

Fig. 1 Integrated components of HBFT partnership training 


\section{Environment and Context}

An HBFT approach utilizes the environment and contexts of the home to assess and treat families. The home provides access to a family's natural setting while the therapist's visit demonstrates a therapist's willingness to enter that environment and access the resources available in the home and surrounding community. The HBFT Partnership assists clinicians in examining ways to adapt treatments to address and incorporate elements of the home environment while offering ways to overcome barriers to service provision.

HBFT creates opportunities for direct translation of therapeutic processes into a family's daily living. The home environment offers the therapist multiple opportunities to experience the family's home life without relying simply on reports of those experiences. However, while the home offers certain advantages, it also brings disadvantages. For instance, while unexpected visitors, phone ringing, or television volume provide data about the family's daily life, they also may disrupt the therapy process. Cherniss and Herzog (1996) note that distractions can be used to empower the family to manage their own boundaries in the midst of competing demands. The HBFT Partnership trainings attempt to guide clinicians to view "distractions" as important data revealing the family's lived experiences and opportunities for direct intervention.

Some have suggested that the therapist's home visit sends an implied message to the family that the therapist is going the extra mile to meet the family and address their issues (Christensen 1995; Woodford et al. 2006). In addition, the therapist has several opportunities to use the immediacy of in-home experiences to help raise the family's awareness of issues and engage the members in specific, change-making behaviors. The HBFT Partnership reminds clinicians about the powerful effect of their efforts to meet the family in their own space as aspects of developing a level of trust with and demonstrating respect for the family.

On another level, home-based work is community-based work. While there are multiple resources within the home that inform therapy, the context of that home in the broader community serves to further broaden the scope of treatment, utilizing available resources such as relationships with neighbors and friends as well as school, church, and civic organizations. Establishing more direct 
connections with these resources may reduce the family's need for formal human service system involvement over time. While HBFT therapists often feel isolated from their supervisors and colleagues, the HBFT Partnership explores ways the clinicians can develop potential partners with those in the family's surrounding community.

\section{Family Roles and Expectations}

An HBFT approach recognizes a family's unique position of familiarity, comfort, and authority in their own home. HBFT is often chosen because other office-based approaches have not been successful. Experiences of the family's roles and expectations in the office can reflect a position of powerlessness and limited investment in therapy. An HBFT approach aims to foster family ownership and influence throughout the treatment process. Family members bring varying concerns and points of view to therapy. Aside from diverse perspectives of the presenting problem, a family approaches therapy with specific role expectations for each family member, perceptions about the therapist's role within their home (Lawson 2005), and an assessment about the role and ability of therapy to address the therapeutic issues. While family members attending therapy in an officebased setting may feel a degree of intimidation, the home visit offers a familiarity that can enhance the members' perception of their active and empowered role within therapy (Fuller 2004). The HBFT Partnership instructs clinicians on ways to invite family members to become involved in the process of therapy, beginning with joining and throughout the phases of treatment. Clinicians are instructed on ways to ask the family members to teach and model for them their family experiences in the home. Families involved in HBFT are often chosen to participate in this form of therapy precisely because they have multiple problems, often caught in cycles of perpetual crises (Adams and Maynard 2000; Cortes 2004; Kagan and Schlosberg 1989; Slesnick and Prestopnik 2004). Brymer and Phillips (2006) and Cortes (2004) suggest that the involvement of social service systems with a family may, over time, create an expectation of the system's ongoing presence. Visiting the family in their home has the potential of inverting roles and expectations and reestablishing the family's place "in the driver seat" of the therapy process and ultimately informing the decisions affecting their lives (Osher and Osher 2002). Training is focused on helping HBFT clinicians to use the family's "host" role to actively participate in treatment. 


\section{Therapist Roles and Expectations}

The HBFT Partnership trainings equip clinicians with the awareness and skills needed to assist them in transforming the home environment from a living space to a therapeutic space. Identifying resources that can inform the therapist's understanding of the family's daily experiences, the therapist more effectively addresses current issues in the context of ongoing family interactions. Training is focused on ways to embrace the "guest" status and enable clinicians to listen, interact, and interpret their experiences in the home. The trainings give attention to the family's culture and, therefore, enable clinicians to hold assumptions and judgments about their experiences tentatively. Therapists are equipped to make the following adaptations: modifying office-based practices to work in the home, adjusting responsibilities associated with being a visitor rather than a host, adapting therapeutic skills that use the additional data offered by the home context, and monitoring the tension between responsibilities associated with work with the family and responsibilities to the agency.

HBFT enables the therapist to learn information in the first visit that may take several sessions of listening to family descriptions in the office. Doing therapy in the home provides a dramatic representation of the family experiences and environment-information the family may not think to report or have words to describe. Direct observations and experiences while interacting with the family in their home provide the therapist familiarity with the family members' experiences (Slattery and Knapp 2003; Snyder and McCollum 1999). However, while the visit provides an opportunity to collect additional data, home-based therapists often experience decreased control of the therapeutic milieu that accompanies their role as a visitor in the home environment. Woodford et al. (2006) and Snyder and McCollum (1999) note that the therapist's guest status, in an unfamiliar environment, challenges the therapist's control and, therefore, perceived level of comfort and safety.

HBFT trainings challenge therapists to appropriately determine their role with the family. Therapists are encouraged to continually compare their own perceptions of their role with the family members' views of and responses to their role. Fouad and Arredondo (2007) suggest that a culturally competent therapist engages in a continual process, both inside and outside of the 
therapeutic environment, seeking ways to improve "awareness of self, knowledge of others, and skills and interventions" (p. 8). The implication of these active efforts suggests that the therapist is ethically obligated to provide a therapeutic process that is responsive to the family and the family's culture. As Lindblad-Goldberg et al. (1998) note,

The greatest clinical challenge is to create, in essence, a wide-angle therapeutic lens that allows the therapist to conduct a comprehensive assessment, and at the same time, a focusing mechanism to zoom in on the key elements requiring change within the defined treatment period. (p. 143)

Cultural competency provides the therapist with the ability necessary to work with the family while adhering to a respectful, curious, and collaborative approach.

\section{Therapeutic Relationship}

Interdisciplinary collaboration at varying levels is a hallmark of HBFT (Saldana and Sheidow 2005; Slattery and Knapp 2003; Stinchfield 2004). The HBFT Partnership was established through collaborations with state and local agencies. The HBFT trainings instruct clinicians on ways to establish collaborative relationships with their clients, other community agencies and resources, as well as their own agency.

Fuller (2004) notes that therapy in the home must transition from socializing and guest-oriented activities to developing a shared treatment focus. The therapist is responsible for guiding the treatment process while remaining responsive to and empowering the family members' participation. The therapist's efforts to refine and strengthen the parental role are especially important since parents are very aware of the threat posed by possible out of home placement for their children. A relationship of mutual respect, trust, and collaboration requires repeated reinforcement of the parents' ability to choose from the available therapeutic options, identifying the parents' existing skills, and developing new ones to meet the current challenges. As the therapist demonstrates ways to collaborate with the family, the family learns the value of 
collaboration and is better prepared, with the therapist's help, to identify and use other resources in the community.

The design and functioning of the HBFT Partnership was established to provide a model for collaboration with the assumption that collaboration at one level has an isomorphic effect of encouraging collaborations to occur at other levels. Trainings focus on ways to establish an effective therapeutic relationship that prioritizes collaboration as an overarching goal of homebased treatment.

\section{Goals of Clinical Work}

Finally, the HBFT Partnership addresses the importance of developing treatment goals that intentionally incorporate the information and resources available in the family's natural environment. Trainings assist clinicians to use overarching principles of contextual goaling that orient the goals toward parent empowerment and increase access to natural supports.

A child's diagnosis(es) and risk for out of home placement are the issues that precipitate a family's referral for HBFT in Kansas. While a diagnostic focus on the child makes financial and social service resources available to the family, the therapeutic focus remains on the family system of which that child is one part (Sheidow and Woodford 2003). Despite the individual designation, the whole family system is addressed. Attention is paid to the ongoing interaction patterns between the members and the available instrumental, emotional, and relational resources they share (Slattery and Knapp 2003; Woolston et al. 1998). Curtis et al. (2004) explain that the treatment is focused on the family relations that serve as the context for the child's behavior with an equally important awareness of the home and community that serve as contexts for the family. Treatment situates the family within larger contexts of extended family, community, social services, and faith communities (Brymer and Phillips 2006; Crenshaw 2004; Woolston et al. 1998; Zarski and Zygmond 1989). Within the family context, attention is given to the parental and sibling roles and the mutually influencing effects of each. Additionally, a focus on empowering parents reflects ways they become vital resources used to facilitate pragmatic changes within the family. 
While HBFT addresses family specific goals, the trainings assist clinicians to achieve meta goals that may lead to ongoing family success. Meta goals focus on equipping families with: increased access to resources, improved generalizability of skills applied to new situations, greater awareness and use of family strengths, and increased awareness and involvement in the community.

\section{Factors Influencing the Decision to Use an HBFT Approach}

As with any therapeutic approach, HBFT models have strengths and limitations. In this section, we address the indications and contraindications for using an HBFT approach. We also address ways the HBFT Partnership assists in the decision-making process to determine when an HBFT approach is appropriate. A decision to use HBFT involves the examination of three different dimensions of clinical work: (1) family presenting issues, (2) historical and current course of treatment, and (3) therapist characteristics and competencies. Determining a "good fit" for using HBFT entails making a determination about the costs and benefits as compared with other treatment modalities. Benefits of utilizing an HBFT approach reflect decreased numbers of foster care placements thus increasing family involvement, preserving the integrity of the family, and limiting the additional psychological and financial costs that are associated with out-of-home placements (Cortes 2004; Fuller 2004; Woodford 1999).

\section{Indications Specific to HBFT}

Families considered for involvement in HBFT tend to have multiple problems, increased risk of the child being placed out of the home, and limited treatment accessibility (Lindblad-Goldberg et al. 1998). The HBFT Partnership trains clinicians to offer novel approaches to address specific clinical issues, overcome barriers to treatment, identify problems arising with previous treatments that have had limited success, and organize family involvement with multiple systems.

Several studies have suggested that a home-based approach has demonstrated reasonable degrees of effectiveness in addressing the following clinical issues: children identified as "seriously emotionally disturbed" or as having a "serious emotional disturbance" (Cherniss and Herzog 1996; Curtis et al. 2004; Fuller 2004; Schmidt et al. 2006; Sexton and Alexander 2000; Stinchfield 2004; 
Woodford 1999; Woolston et al. 1998; Zarski et al. 1992; Zarski and Fluharty 1992); children and adolescents exhibiting antisocial behaviors, juvenile delinquents, and those involved with Juvenile Justice Services (Cherniss and Herzog 1996; Curtis et al. 2004; Sexton and Alexander 2000; Woodford 1999; Woolston et al. 1998; Zarski et al. 1992; Zarski and Fluharty 1992; Zarski and Zygmond 1989); children with autism (Cottrell 1994); children in need of foster care (Fuller 2004); and families more broadly defined as multiproblem, at-risk, or multi-challenged (Adams and Maynard 2000; Cortes 2004; Johnson et al. 2002; Schacht et al.1989; Slattery and Knapp 2003; Snyder and McCollum 1999; Zarski and Zygmond 1989). Each of these articles has revealed that families involved in HBFT have experienced greater benefits than those who engaged in traditional, office-based treatment approaches. Results have included member symptom relief, improved family interactions, and families' increased awareness and access to community resources.

Some also have suggested that HBFT approaches provide a way to overcome various physical, psychological, emotional, and cultural barriers to treatment. Families who experience physical barriers often lack transportation, live a considerable distance from a mental health agency, or include a family member who has physical conditions limiting mobility. Visiting the home increases access to and inclusion of family members who might not otherwise attend therapy in the office (Cortes 2004; Cottrell 1994; Schacht et al. 1989; Woodford 1999; Zarski et al. 1991; Zarski and Zygmond 1989). Psychological and emotional barriers are reflected when a key family member resists attending treatment in the office (Schacht et al. 1989; Zarski and Fluharty 1992; Zarski and Zygmond 1989), or when family members fear and distrust social services and assume that attending therapy in an office may jeopardize the family's perceived security (e.g., immigration status). Cultural barriers often relate to a family's conceptualization about family boundaries. An example occurs when family boundaries are closed, suggesting that a member should turn toward the family for help rather than away from the family to seek help outside of the family system. Families with rigid boundaries may overcome the cultural barrier to treatment if they perceive a therapist's visit to the family home as relieving them of having seek help outside but may simultaneously feel challenged while allowing the therapist into the family milieu.

An HBFT approach is often warranted when a family has experienced limited or no change resulting from past treatment efforts employing other treatment modalities (Schmidt et al. 2006). 
Furthermore, HBFT has been indicated when a member has not improved after a series of hospitalizations (Schacht et al. 1989; Zarski et al. 1992; Zarski and Zygmond 1989), his or her condition is directly associated with family interactional patterns, or changes have not resulted in a sustainable resolution of the presenting problems (Fuller 2004). HBFT treatment also has been indicated when family members have difficulty generalizing learning and experiences from treatment interventions to daily living outside of the treatment setting (Woods 1988).

Families involved with multiple systems may experience additional support when an HBFT approach facilitates ongoing consultations and collaborations between those systems. Families involved in multiple systems often experience stresses associated simply with the time and involvement of interacting with the differing agencies, including coordinating multiple appointment schedules, problem-solving transportation issues, and taking time off from work. These stressors are further exacerbated when the family is faced with responding to and resolving competing expectations. The cross-system coordination of services establishes compatible resourcing and scheduling to meet the family needs.

The HBFT Partnership trains clinicians to foster a therapeutic relationship that utilizes the home environment, identify family roles and expectations, and assess barriers to treatment using a culturally informed approach. The one-day training leads clinicians through an hour and a half experiential exercise exploring cultural differences; reviewing implications for referrals, assessment, and treatment; and examining the relationship of cultural issues and self-of-thetherapist issues.

\section{Contraindications Specific to HBFT}

A thorough review of the literature todate revealed very few explicit contraindications for using an HBFT approach. Those that are mentioned tend to apply to therapy in general and family therapy in particular. The issues raised reveal potential concerns for using an HBFT approach when families give evidence of the following: active abuse, violence, or neglect; acute psychiatric or medical crises or untreated substance abuse; negative perceptions of treatment; and repeated unsuccessful HBFT treatments. An additional factor that potentially contraindicates using an HBFT approach 
occurs when a therapist lacks an appropriate skill level for managing the additional challenges of providing therapy in the home environment.

Family therapy offered in the office or the home has been contraindicated when family members are at risk of exposure to emotional or physical abuse, violence, or neglect within the context of those relationships. Chaffin (2006), Epstein (1997), and Littell (1997) examined the results of family preservation services and suggested that there is limited evidence of long-term effectiveness of addressing those issues. They each suggest the need for additional rigorous studies that evaluate the process of treatment to determine the mechanisms that lead to sustainable outcomes. Treatments involving families presenting with abuse or neglect potentially jeopardize the safety of the least powerful members. HBFT under those circumstances also may present safety risks to the therapist who lacks immediate access to supervision and other supports (Cortes 2004; Fuller 2004). Therapist safety in the home is often compromised by other specific hazards such as aggressive dogs, no cell phone coverage, and the potential threat of angry clients (Christensen 1995).

Family therapy that is provided without first addressing a family member's acute medical or psychiatric crises or untreated substance abuse could lead to further instability, thus rendering therapeutic efforts ineffective. Family therapy has repeatedly been acknowledged as an effective modality to work with families with a member who is abusing a substance (Becker and Curry 2008; Henggler et al. 1996; Rowe and Liddle 2003). However, HBFT would be contraindicated if it simply focuses on the substance-abusing member (Steinglass 2009), or if that member is actively using substances and resists addressing the impact of his or her substance abuse or dependence on the family. Working with the family system while at least one member remains substance-impaired may result in unduly burdening the other members with an implied message that suggests they take complete responsibility for the change process. This work also raises potential safety risks for the other family members and the therapist visiting the home.

A review of a family's past or present course of treatment may reveal reasons that preclude using an HBFT approach. Such reasons include demonstrations of unresolved issues, involvement in perpetual cycles of crises, exhibiting repeated resistance to or absence from treatment, or continued displays of a distractive and chaotic home environment. The first three family issues often precede 
an HBFT referral. These issues reflect unaltered family patterns that have rigidified over time and can appear resistant to clinical efforts. When family members have integrated these patterns despite repeated involvement with in-office treatment, it is reasonable to suggest that the prior treatment approaches actually may have reinforced the family members' responses. These three enduring conditions signal the difficulties inherent in much of the work referred for HBFT. Furthermore, if the family referred for HBFT also perceives the additional efforts as simply more in a long series of ineffective and imposing experiences, then an HBFT approach may also be ineffective.

Families often enter into a series of HBFT treatments involving many different therapists. A family demonstrating little or no evidence of success despite previous intensive home-based treatments suggests either (1) the treatment approach has not been appropriately matched and responsive to the family, or (2) the family has not reached a level of readiness to change needed to engage in additional home-based work (Prochaska and Norcross 1999). Families with these experiences may demonstrate their dissatisfaction with or a lack of buy-into the treatment process through repeated absences when a therapist arrives at the home for a session or active resistance to treatment interventions. These responses suggest that the current contract for treatment requires review, adjustment, and perhaps, reconsideration of the provision of HBFT at the current time.

The remaining issue that may contraindicate using an HBFT approach reflects potential gaps in the skill level and training of clinicians attempting to utilize the home environment. A therapist's ability to guide treatment while navigating through the unique challenges of HBFT requires specialized skills that are often missing from graduate training programs (Cortes 2004; Lawson and Foster 2005). Those skills include: deliberate and integrated use of the home environment in developing goals and interventions; adapting treatment to involve children, adults, and the whole family system; managing proper professional boundaries that honor the distinctions among family member and clinician roles and responsibilities; effectively transforming the "home visit" into therapeutic work; and matching services to the family needs. The increasing difficulty and intensity of working with families in their home requires the clinician to develop specialized skills. Lawson and Foster (2005) suggest that "In situations such as home-based counseling, where the treatment environment is unstructured, the counselor must be functioning at a higher conceptual level for the counseling interactions to be effective" (p.155). When those skills are absent or 
underdeveloped there is a potential to undermine HBFT treatment effectiveness and reify a family's negative perceptions concerning the value and efficacy of therapy (Cortes 2004; Lawson and Foster 2005).

HBFT trainings assist clinicians in decision-making and equipping processes needed to determine whether HBFT is the appropriate modality for use with particular families. The trainings help clinicians to reflect upon their existing skills and identify additional skills needed to meet the demands of an HBFT approach. The trainings encourage exploration and skill development to enable clinicians to develop plans that insure adequate safety, utilize supervision and collegial support, identify areas needed for additional training, and establish an ongoing program of selfcare.

\section{Conclusion}

The HBFT Partnership was developed to meet the demand for a statewide dissemination of training and support of HBFT therapists designed to meet the unique challenges of home-based work in varying contexts. Process and outcome evaluations are being developed to examine the effectiveness of individual components of the partnership. These evaluations will assess the effectiveness of the training approaches, the relevance of the topics, and the impact of clinicians' participation in the various partnership components on their work. The relationships among the following variables will also be examined: therapist levels of clinical experience and training, therapist learning, and the impact of varying strategies of self-care and supervision on treatment effectiveness. Outcome evaluations will compare the training data with the data available from state agencies reflecting the frequency of families' use, types, and extent of therapeutic services.

The continued development of the HBFT Partnership faces significant challenges inherent to collaborating with diverse agencies and clinicians from various disciplines. However, focusing on the common components of HBFT has enabled us to develop trainings with a broad appeal and application. The ongoing emphasis on collaboration has enabled us to become aware of that diversity while tailoring our approaches to meet the varied training needs. 


\section{References}

Adams, J., \& Maynard, P. (2000). Evaluating training needs for home-based family therapy: A focus group approach. The American Journal of Family Therapy, 28, 41-52.

Becker, S., \& Curry, J. (2008). Outpatient interventions for adolescent substance abuse: A quality of evidence review. Journal of Consulting and Clinical Psychology, 76(4), 531-543.

Berg, I. (1994). Family-based services: A solution-focused approach. New York: Norton.

Boyd-Franklin, N., \& Bry, B. (2000). Reaching out in family therapy: Home-based, school, and community interventions. New York: Guilford.

Bronfenbrenner, U. (1979). The ecology of human development: Experiments by nature and design. Cambridge, MA: Harvard University Press.

Brymer, L., \& Phillips, J. (2006). Contextual meanings in action: Integration of social work and family therapy in child protective services. Journal of Systemic Therapies, 25(2), 13-23.

Chaffin, M. (2006). The changing focus of child maltreatment research and practice within psychology. Journal of Social issues, 62(4), 663-684.

Chamberlain, P., \& Smith, D. K. (2003). Antisocial behavior in children and adolescents: The Oregon multidimensional treatment foster care model. In A. Kazdin \& G. Weisz (Eds.), Evidence-based psychotherapies for children and adolescents. New York: Guilford.

Cherniss, C., \& Herzog, E. (1996). Impact of home-based family therapy on maternal and child outcomes in disadvantaged adolescent mothers. Family Relations, 45, 72-79.

Christensen, L. (1995). Therapists' perspectives on home-based family therapy. The American Journal of Family Therapy, 23(4), 306-314.

Cortes, L. (2004). Home-based family therapy: A misunderstanding of the role and a new challenge for therapists. The Family Journal: Counseling and Therapy for Couples and Families, 12(2), 184-188.

Cottrell, D. (1994). Family therapy in the home. Journal of Family Therapy, 16, 189-197.

Crenshaw, W. (2004). Treating families and children in the child protective system: Strategies for systemic advocacy and family healing. New York: Brunner-Routledge.

Curtis, N., Ronana, K., \& Borduin, C. (2004). Multisystemic treatment: A meta-analysis of outcome studies. Journal of Family Psychology, 18(3), 411-419.

Epstein, W. (1997). Social science, child welfare, and family preservation: A failure of rationality in public policy. Children and Youth Services Review, 19(1/2), 41-60. 
Figley, C. (Ed.). (2002). Treating compassion fatigue. New York: Routledge.

Fouad, N., \& Arredondo, P. (2007). Becoming culturally oriented: Practical advice for psychologists and educators. Washington, D.C.: American Psychological Association.

Fraser, M., \& Haapala, D. (1987). Home-based family treatment: A quantitative-qualitative assessment. The Journal of Applied Social Sciences, 12(1), 1-23.

Fuller, A. (2004). Crisis: Home-based family therapy. Australian and New Zealand Journal of Family Therapy, 25(4), 177-182.

Henggeler, S., \& Lee, T. (2003). Multisystemic treatment of serious clinical problems. In A. Kazdin \& J. Weisz (Eds.), Evidence-based psychotherapies for children and adolescents. New York: Guilford.

Henggler, S., Pickrel, S., Brondino, M., \& Crouch, J. (1996). Eliminating (almost) treatment dropout of substance abusing or dependent delinquents through home-based multisystemic therapy. The American Journal of Psychiatry, 153(3), 427-428.

Institute for Family Development. (2005). Welcome to the Institute for Family Development developers of the Homebuilders program. Retrieved January 8, 2008, from http://www.institutefamily.org/default.asp.

Johnson, L., Wright, D., \& Ketring, S. (2002). The therapeutic alliance in home-based family therapy: Is it predictive of outcome? Journal of Marital and Family Therapy, 28(1), 93-102.

Kagan, R., \& Schlosberg, S. (1989). Families in perpetual crisis. New York: Norton.

Lawson, G., \& Foster, V. (2005). Developmental characteristics of home-based counselors: A key to serving at-risk families. The Family Journal: Counseling and Therapy for Couples and Families, 13(2), 153-161.

Lindblad-Goldberg, M., Dore, M., \& Stern, L. (1998). Creating competencies from chaos: A comprehensive guide to home-based services. New York: Norton.

Littell, J. (1997). Effects of the duration, intensity, and breadth of family preservation services: A new analysis of data from the Illinois Family First Experiment. Children and Youth Services Review, 19(1/2), 17-39.

National Institute of Mental Health. (2007). Alliance for Research Progress Report. Washington, D.C.: National Institute of Mental Health.

Osher, T., \& Osher, D. (2002). The paradigm shift to true collaboration with families. Journal of Child and Family Studies, 11(1), 47-60. 
Prochaska, J., \& Norcross, J. (1999). Systems of psychotherapy: A transtheoretical analysis. Pacific Grove, CA: Brooks/Cole.

Rowe, C., \& Liddle, H. (2003). Substance abuse. Journal of Marital and Family Therapy, 29(1), $97-120$.

Saldana, L., \& Sheidow, A. (2005). Multisystemic therapy: Origins, empirical results, and current efforts. Family Therapy Magazine, 4, 26-29.

Schacht, A., Tafoya, N., \& Mirabla, K. (1989). Home-based therapy with American Indian families. The Journal of the National Center, 3(2), 27-42.

Schmidt, M., Lay, B., Gopel, C., Naab, S., \& Blanz, B. (2006). Home treatment for children and adolescents with psychiatric disorders. European Child and Adolescent Psychiatry, 15(5), $265-276$.

Sexton, T., \& Alexander, J. (2000). Functional family therapy. Washington, D.C.: U.S. Department of Justice.

Sheidow, A., \& Woodford, M. (2003). Multisystemic therapy: An empirically supported, homebased family therapy approach. The Family Journal: Counseling and Therapy for Couples and Families, 11(3), 257-263.

Slattery, J., \& Knapp, S. (2003). In-home family therapy and wraparound services for working with seriously at-risk children and adolescents. In L. VandeCreek \& T. Jackson (Eds.), Innovations in clinical practice: Focus on children \& adolescents. A volume in the innovations in clinical practice series (Vol. viii, pp. 135-149). Sarasota, FL: Professional Resource Press/Professional Resource Exchange.

Slesnick, N., \& Prestopnik, J. (2004). Office versus home-based family therapy for runaway, alcohol abusing adolescents: Examination of factors associated with treatment attendance. Alcoholism Treatment Quarterly, 22(2), 3-19.

Snyder, W., \& McCollum, E. (1999). Their home is their castle: Learning to do in-home family therapy. Family Process, 38, 229-242.

Stamm, B. (2002). Professional quality of life: Compassion satisfaction and fatigue subscales-III. From http://www.isu.edu/ bhstamm.

Steinglass, P. (2009). Systemic-motivational therapy for substance abuse disorders: An integrative model. Journal of Family Therapy, 31, 155-174.

Stinchfield, T. (2004). Clinical competencies specific to family-based therapy. Counselor Education and Supervision, 43, 286-300. 
United States Congress. (1993). Family preservation and support services program act of 1993 (No. P.L. 103-66). Washington, DC: United States Congress.

Wasik, B., \& Roberts, R. (1994). Home visitor characteristics, training, and supervision: Results of a national survey. Family Relations, 43(3), 336-341.

Woodford, M. (1999). Home-based family therapy: Theory and process from "friendly visitors" to multisystemic therapy. The Family Journal: Counseling and Therapy for Couples and Families, 7(3), 268-269.

Woodford, M., Bordeau, W., \& Alderfer, C. (2006). Home-based service delivery: Introducing family counselors in training to the home as a therapeutic milieu. The Family Journal: Counseling and Therapy for Couples and Families, 14(3), 240-244.

Woods, L. (1988). Home-based family therapy. Social Work, 33(3), 211-214.

Woolston, J., Berkowitz, S., Schaefer, M., \& Adnopoz, J. (1998). Intensive, integrated, in-home psychiatric services: The catalyst to enhancing outpatient intervention. Child and Adolescent Psychiatric Clinics of North America, 7(3), 615-632.

Zarski, J., Aponte, H., Bixenstine, C., \& Pamela, C. (1992). Beyond home-based family intervention: A multi-family approach toward change. Contemporary Family Therapy, $14(1), 3-14$.

Zarski, J., \& Fluharty, L. (1992). Treating emotionally disturbed youth: A comparison of homebased and outpatient interventions. Contemporary Family Therapy, 14(4), 335-350.

Zarski, J., Greenbank, M., Sand-Pringle, C., \& Cibik, P. (1991). The invisible mirror: In-home family therapy and supervision. Journal of Marital and Family Therapy, 17(2), 133-143.

Zarski, J., \& Zygmond, M. (1989). Negotiating transitions: A supervision model for home-based family therapists. Contemporary Family Therapy, 11(2), 119-130. 